\title{
Magneto-Gyrotropic Effect in Semiconductor Quantum Wells
}

\author{
S.D. Ganichev, V.V. Bel'kov*, Petra Schneider, S. Giglberger, S.N. Danilov, \\ W. Weber, M. Olteanu, and W. Prettl \\ Universität Regensburg, D 93040 Regensburg, Germany
}

\begin{abstract}
A new type of infrared induced photocurrents is reported occurring in gyrotropic quantum wells in the presence of a magnetic field. The effect is caused by spin-orbit interaction and may be observed even for unpolarized radiation. The current occurs for particular relative orientation between magnetic field, current and crystallographic orientation.
\end{abstract}

\section{Introduction}

The photogalvanic effect (PGE) in semiconductors is characteristic for gyrotropic materials and was recently intensively studied, both theoretically and experimentally, in zinc-blende and diamond-lattice quantum well (QW) structures $[1,2]$. In such systems a photocurrent flows under illumination with circularly polarized light which changes its direction if the helicity of the circular polarization is reversed. However, in the presence of a magnetic field a photocurrent can flow even if the light is unpolarized $[3,4]$. This will be denoted as magneto-gyrotropic PGE below. The effect is due to the fact that the gyrotropic point-group symmetry makes no difference between polar and axial vectors and therefore currents $j \propto I B$ are allowed by symmetry with $I$ the light intensity, $B$ the applied magnetic field an invariant proportionality constant. The magnetogyrotropic PGE has been studied theoretically in bulk crystals and nanostructures and was observed in gyrotropic QWs. A detailed list of references is given in [5]. So far the magneto-gyrotropic photocurrent has been observed for.direct optical transitions. Here_we re= port on the observation of the magneto-gyrotropic photocurrent in $n$-doped InAs QWs under indirect intrasubband transitions (Drude-like absorption) of linearly and circularly polarized far-infrared radiation at photon energies smaller than the subband separation.

\section{Experimental Technique}

The experiments were carried out at room temperature on (001)-oriented $n$-type InAs/ $\mathrm{Al}_{0.3} \mathrm{Ga}_{0.7} \mathrm{Sb}$ heterostructures having $C_{2 v}$ point symmetry. Single QWs of $15 \mathrm{~nm}$ width with free carrier densities of about $1.3 \cdot 10^{12} \mathrm{~cm}^{-2}$ and mobility $\approx 2 \cdot 10^{4} \mathrm{~cm}^{2} /(\mathrm{Vs})$ were investigated. The samples have four pairs of opposite ohmic contacts, see insets in Figs. 1 (a) - (d). An external magnetic field $B$ up to $1 \mathrm{~T}$ was applied in the plane of the QWs.

A pulsed optically pumped far-infrared $\mathrm{NH}_{3}$ laser was used for optical excitation. The samples were irradiated along the growth direction by linearly or circularly polarized radiation of helicity $P_{c i r c}$. In all experiments the electric field vector of linearly polarized radiation was oriented perpendicularly to the magnetic field. The photocurrent $j$ was measured in unbiased structures as the voltage drop across a $50 \Omega$ load resistor in a closed circuit configuration.

\section{Experimental Results}

By irradiating the QWs with normal incident linearly polarized light a photocurrent signal has been detected after applying an in-plane magnetic field. The polarity of the current changes upon reversal of the magnetic field. Measurements are presented for radiation with $\lambda=148 \mu \mathrm{m}$. For $B$ aligned along a $\langle 110\rangle$ axis only a current flow perpendicular to the applied magnetic field was detected (triangles in Figs. 1 (a) and (b)). For another experimental configuration, $B \|\langle 100\rangle$, both longitudinal and transverse currents were observed shown in Fig. 1 (c). In the absence of a magnetic field the signals vanish for all directions.

A magnetic field induced current has also been observed upon excitation with circularly polarized radiation yielding a current in both directions, normal and parallel to the magnetic field (Figs. 1 (a) and (b)). The current component normal to the magnetic field, ap-plied along $y^{\prime} \|[110]$, is independent of the radiation helicity and coincides with that induced by linearly polarized radiation, say $j_{x^{\prime}} \propto I B_{y^{\prime}}$. This observation indicates that the origin of this current is the same for both linearly and circularly polarized light. In contrast, the current along the magnetic field direction changes its sign upon changing the helicity from right- to lefthanded and vanishes for linearly polarized radiation: $j_{y^{\prime}} \propto I B_{y^{\prime}} P_{\text {circ }}$ (see Fig. 1 (b)). This current is caused by the spin-galvanic effect [6].

\section{Discussion}

The magnetic field induced photogalvanic effect can phenomenologically be derived by group theory. The present experiments have been carried out on (001)oriented QWs of $C_{2 v}$ point symmetry. In the coordinate 

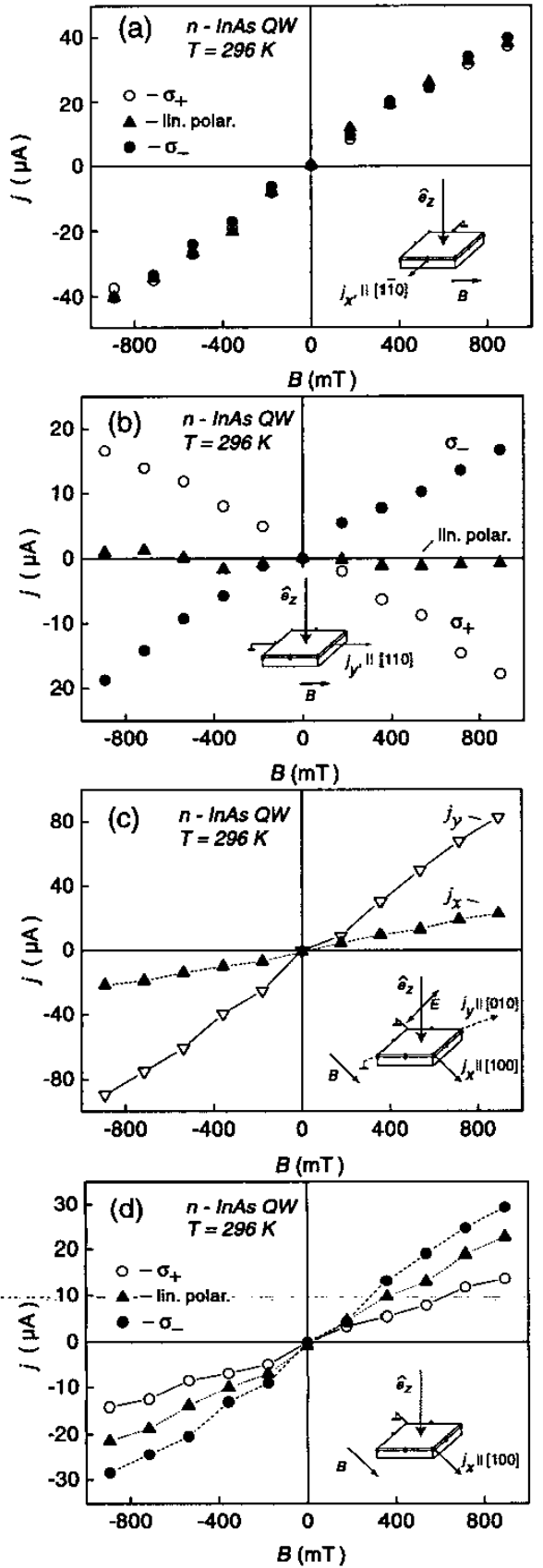

Figure 1: Magnetic-field dependence of the photocurrent measured with magnetic field $\boldsymbol{B}$ parallel to ((a) and (b)) [110] direction and parallel to ((b) and (c)) [100]. Pulsed optical excitation of $10 \mathrm{~kW}$ power at normal incidence was applied at wavelength $\lambda=148 \mu \mathrm{m}$ with linear, right-handed circular $\left(\sigma_{+}\right)$, and left-handed circular $\left(\sigma_{-}\right)$polarization. The current is measured (a) normal to $B$, (b) and (d) parallel to $B$, (c) normal and parallel to $B$. The insets show the geometry of the experiments. system $x^{\prime}\left\|[1 \overline{1} 0], y^{\prime}\right\|[110], z \|[$ [001], the photocurrent can be written as

$$
\begin{gathered}
j_{x^{\prime}}=S_{1} B_{y^{\prime}} I+S_{2} B_{y^{\prime}}\left(\left|e_{x^{\prime}}\right|^{2}-\left|e_{y^{\prime}}\right|^{2}\right) I+ \\
S_{3} B_{x^{\prime}}\left(e_{x^{\prime}} e_{y^{\prime}}^{*}+e_{y^{\prime}} e_{x^{\prime}}^{*}\right) I+S_{4} B_{x^{\prime}} I P_{\text {circ }} \\
j_{y^{\prime}}=S_{1}^{\prime} B_{x^{\prime}} I+S_{2}^{\prime} B_{x^{\prime}}\left(\left|e_{x^{\prime}}\right|^{2}-\left|e_{y^{\prime}}\right|^{2}\right) I+ \\
S_{3}^{\prime} B_{y^{\prime}}\left(e_{x^{\prime}} e_{y^{\prime}}^{*}+e_{y^{\prime}} e_{x^{\prime}}^{*}\right) I+S_{4}^{\prime} B_{y^{\prime}} I P_{\text {circ }}
\end{gathered}
$$

The parameters $S_{i}$ and $S_{i}^{\prime}(i=1 \ldots 4)$ are invariants and $e$ is the radiation polarization vector. In terms of this equations the experimental results can qualitatively be described. The equations show that the first terms on the right hand side yield a current in the plane of the QW for polarized as well as for unpolarized radiation. The second and third terms assume a maximum for linear polarization and vanish for circular polarization. The last terms describe a current proportional to the helicity of radiation. At zero magnetic field no current in response to irradiation, neither linearly nor circularly polarized, is allowed by symmetry in agreement to the experiment. On a microscopic level the observed magneto-gyrotropic PGE can be attributed to three possible mechanisms. At first the electric current appears due to an asymmetry of spin-dependent spinconserving energy relaxation processes in a system of hot carriers heated by free carrier absorption [4]. Another mechanism is based on the asymmetry of spinflip processes and represents in fact the spin-galvanic effect [6]. Finally a diamagnetic mechanism yielding spin-independent magnetic field induced $\boldsymbol{k}$-linear terms in the electron Hamiltonian of the type $\propto\left(B_{x} k_{y}-B_{y} k_{x}\right)$ may also contribute to a photocurrent.

Financial support by the DFG is gratefully acknowledged.

*Permanent address: A.F. Ioffe Physico-Technical Institute, 194021 St. Petersburg, Russia

\section{References}

[1] E.L. Ivchenko, Usp. Fiz. Nauk 45, 1461 (2002) [Phys. Uspekhi 45, 1299 (2002)].

[2] S.D. Ganichev and W. Prettl, J. Phys.: Condens. Matter 15, 935 (2003).

[3] E.L. Ivchenko and G.E. Pikus, Pis'ma Zh. Eksp. Teor. Fiz. 27, 640 (1978) [JETP Lett. 27, 604 (1978).

[4] E.L. Ivchenko and G.E. Pikus, Izv. Akad. Nauk SSSR (ser. fiz.) 47, 2369 (1983) [Bull. Acad. Sci. USSR, Phys. Ser., 47, 81 (1983)].

[5] V.V. Bel'kov, S.D. Ganichev, Petra Schneider, S. Giglberger, E.L. Ivchenko, S.A. Tarasenko, W. Wegscheider, D. Weiss, and W. Prettl, arXiv:cond-mat/0311474 v1.

[6] S.D. Ganichev, E.L. Ivchenko, V.V. Bel'kov, S.A. Tarasenko, M. Sollinger, D. Weiss, W. Wegscheider, and W. Prettl, Nature (London) 417, 153 (2002). 